\title{
Flavonoid Derivatives for Monoamine Oxidase-A Inhibition
}

\author{
Arman N. Esfahani ${ }^{1}$, Mahmoud Mirzaei $^{2, 凶}$
}

Received: 15 December 2019 / Accepted: 18 December 2019 / Published Online: 18 December 2019

(C) SAMI Publishing Company (SPC) 2019

\section{ABSTRACT}

The in silico molecular docking (MD) simulations have been performed to examine the efficacy of three flavonoid ligands including chrysin, apigenin and luteolin on monoamine oxidase-A (MAO-A) enzyme inhibitions in comparison with the reference moclobemide inhibitor. All the obtained quantitative and qualitative results indicated that the flavonoid ligands could be proposed as possible inhibitors for MAOA enzyme activity. The most important note is that the ligands could interact with the coenzyme of MAO$A$, which is dominant for enzyme inhibition. The results indicated that luteolin could be proposed as the best choice of MAO-A enzyme inhibitor among the investigated ligands.

Keywords: Flavonoid · Monoamine oxidase-A · in silico $\cdot$ Molecular docking $\cdot$ Depression

\section{Introduction}

Monoamine oxidase-A (MAO-A) enzyme is one of the most important leading compound in appearing depression disorder [1]. The containing flavin adenine dinucleotide (FAD) could catalyze the oxidative activity of MAO-A, especially in the major depression disorders [2]. Therefore, inhibiting MAO-A activity could yield proper pharmacotherapy treatments against depression disorder [3]. It is well known that the natural products, as the initial resource of pharmacotherapy, could play dominant roles for medication of several diseases and disorders [4]. Therefore, either extracting them from plants or synthesizing their derivatives is crucial for modern

\footnotetext{
$\triangle$ Corresponding author.

E-mail address: mdmirzaei@pharm.mui.ac.ir (M. Mirzaei)

${ }^{1}$ Isfahan Pharmacy Students' Research Committee, School of Pharmacy and Pharmaceutical Sciences, Isfahan University of Medical Sciences, Isfahan, Iran
}

pharmacotherapy treatments [5]. Flavonoids are among the natural products, in which their antioxidant and anti-inflammatory activities have been already examined based on experiments [6]. Moreover, the synthetic flavonoids could also be considered for the medical treatments [7]. Based on the expected ant-oxidant activity, MAO-A enzyme inhibition of flavonoids have been investigated in this work based on the in silico approach. In addition to the in vitro and in vivo studies, in silico approach could yield detailed information about the mechanism of complicated biological systems [8-16]. For MAO-A inhibition, interaction of ligand with the FAD coenzyme is very much important to ban the enzyme for

\footnotetext{
2 Biosensor Research Center, School of Advanced Technologies in Medicine, Isfahan University of Medical Sciences, Isfahan, Iran
} 
further oxidization [17]. The possibility of ligand for chelating FAD could be very well recognized based on the in silico approach to propose the corresponding ligand for further considerations. Therefore, it is very much important to know details of complicated biological systems based on the in silico approach for making a better judgment about their activities [18]. Within this work, the efficacy of three flavonoid ligands (Table 1) on MAO-A enzyme inhibition are carefully investigated by the in silico approach (Table 2 and
Fig. 2). Moreover, the results are compared with the reference moclobemide, as the known selective inhibitor of MAO-A enzyme [19]. The inhibitions of MAO-enzyme with natural and synthetic products have been earlier investigated based on several employed methodologies; however, there is still no efficient inhibitor for this enzyme yet and further investigations are required [20]. Therefore, this work tries to propose a possible inhibitor of MAO-A to be used possibly for mood disorders in future.

Table 1: Flavonoid derivatives

\begin{tabular}{|c|c|c|c|c|}
\hline & $\mathrm{OH}$ & & & \\
\hline ChemSpider ID & Name & R1 & $\mathbf{R 2}$ & R3 \\
\hline 4444926 & Chrysin & $\mathrm{OH}$ & $\mathrm{H}$ & $\mathrm{H}$ \\
\hline 4444100 & Apigenin & $\mathrm{OH}$ & $\mathrm{H}$ & $\mathrm{OH}$ \\
\hline 4444102 & Luteolin & $\mathrm{OH}$ & $\mathrm{OH}$ & $\mathrm{OH}$ \\
\hline
\end{tabular}

\section{Materials and Methods}

First, 3D structures of three flavonoid ligands including chrysin, apigenin and luteolin (Table 1) and the reference moclobemide (Fig.1) have been obtained from the ChemSpider bank [21] and used as is in this work. Second, 3D structure of MAO-A enzyme has been obtained from the Protein Data Bank [22] with the ID 2BXR and it has been prepared as receptor for Molecular Docking (MD) simulation.

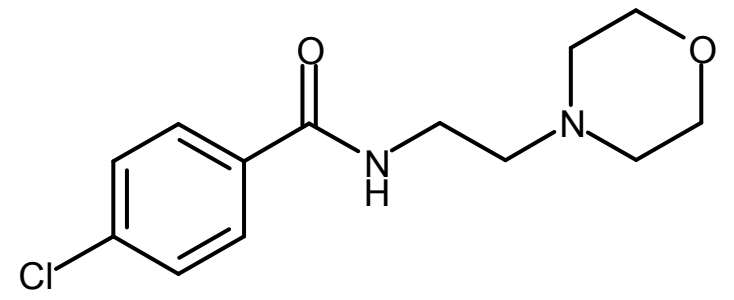

Fig. 1. Moclobemide (ChemSpider ID: 4087).
Subsequently, all ligand and receptor structures have been prepared for MD simulations by the AutoDock-Tools [23] and then the MD simulations have been performed by the AutoDock package [23]. It is worth to note that the obtained results by the MD simulations are very much dependent on the grid-box center and sizes, number of conformation search-runs, employed algorithm and some more factors such as ligand characteristics. For this work, the grid-box size has been set to $70 * 70 * 70$ and the genetic algorithm with 200 search-runs has been employed for MD simulations. The obtained quantitative parameters including binding energy (BE) and inhibition constant (IK) are summarized in Table 2 in addition to the qualitative interacting amino acids (AA). The graphical representations of ligand-receptor complexes are exhibited in Fig. 2. 
Table 2: Molecular Docking Properties*

\begin{tabular}{lllll}
\hline Property & Chrysin & Apigenin & Luteolin & Moclobemide \\
\hline BE kcal/mol & -7.83 & -7.86 & -8.43 & -7.95 \\
IK nM & 1830 & 1750 & 659 & 1490 \\
H-Bonds & GLY71, GLN74, & PRO72, ASN181, & PRO72, THR73, & GLN74, ARG206, \\
AA & ARG206, SER209 & FAD600 & GLN74, ASN181, & GLU216 \\
& & & FAD600 & \\
Other-Bonds & TYR69, VAL70, & TYR69, VAL70, & TYR69, GLY71, & TYR69, VAL70, \\
AA & ILE207, PHE208, & GLY71, THR73, & ILE180, ARG206, & GLY71, PRO72, \\
& GLU216, PHE352, & GLN74, ILE180, & ILE207, PHE208, & THR73, SER209, \\
& TYR407, TRP441, & ARG206, ILE207, & SER209, GLU216, & PHE352, TYR407, \\
& TYR444, FAD600 & PHE208, SER209, & TYR407, TRP441, & TRP441, TYR444, \\
& & GLU216, TRP441, & TYR444 & FAD600
\end{tabular}

*See Fig. 2 for graphical representations.

\section{Results and Discussion}

Within this work, MAO-A enzyme inhibition by three flavonoid derivatives including chrysin, apigenin and luteolin (Table 1) have been investigated based on the in silico MD simulations $[23,24]$. The ligand structures are different from each other based on the existence of hydroxyl groups as indicated in Table 1 . The quantitative results of MD simulations (Table 2 ) could show the effects of structural differences of ligands on their binding strengths with receptor, in which increasing the number of hydroxyl groups yielded increasing in the binding strength by obtaining a better BE for luteolin with three hydroxyl groups. Comparing the results with the reference moclobemide could indicate that only luteolin could compete with moclobemide for a better interaction with the MAO-A receptor. The values of IK are also supporting the obtained BE values for the interacting complex systems of ligandreceptor. Examining the interacting AAs with ligands could show different achievements, in which all three flavonoid ligands interacting with FAD coenzyme through hydrogen bonds $(\mathrm{H}-$ Bonds). However, examining interacting AAs of moclobemide shows that the ligand interacts with FAD of enzyme in other bonds rather than $\mathrm{H}-$
Bonds. It could be remembered here that the advantage of a good ligand for inhibiting MAO-A activity is its interacting with FAD coenzyme, in which they are very well recognized here for all three flavonoid derivatives even better than the reference moclobemide. It is worth to note that the quantitative properties are important to be considered but after examining the qualitative properties for the ligand-receptor interacting systems, as could be seen by the investigated ligands versus the reference ligand. Comparing again the flavonoid ligands with the reference moclobemide regarding their qualitative AAs interactions could show that the investigated ligands could be proposed for the MAO-A enzyme inhibition, in which all of them interact with FAD coenzyme through $\mathrm{H}$-Bonds. However, the luteolin ligand with three additional hydroxyl groups could be still the best choice among the three ligands in either quantitative or qualitative aspects. As a concluding remark, the flavonoid ligands could inhibit the MAO-A activity, especially with more functionalized hydroxyl groups to the core ligand structure. The in silico approach very well recognized the mechanism of interactions of ligands with the enzyme to prepare clear achievements about the work purpose. 

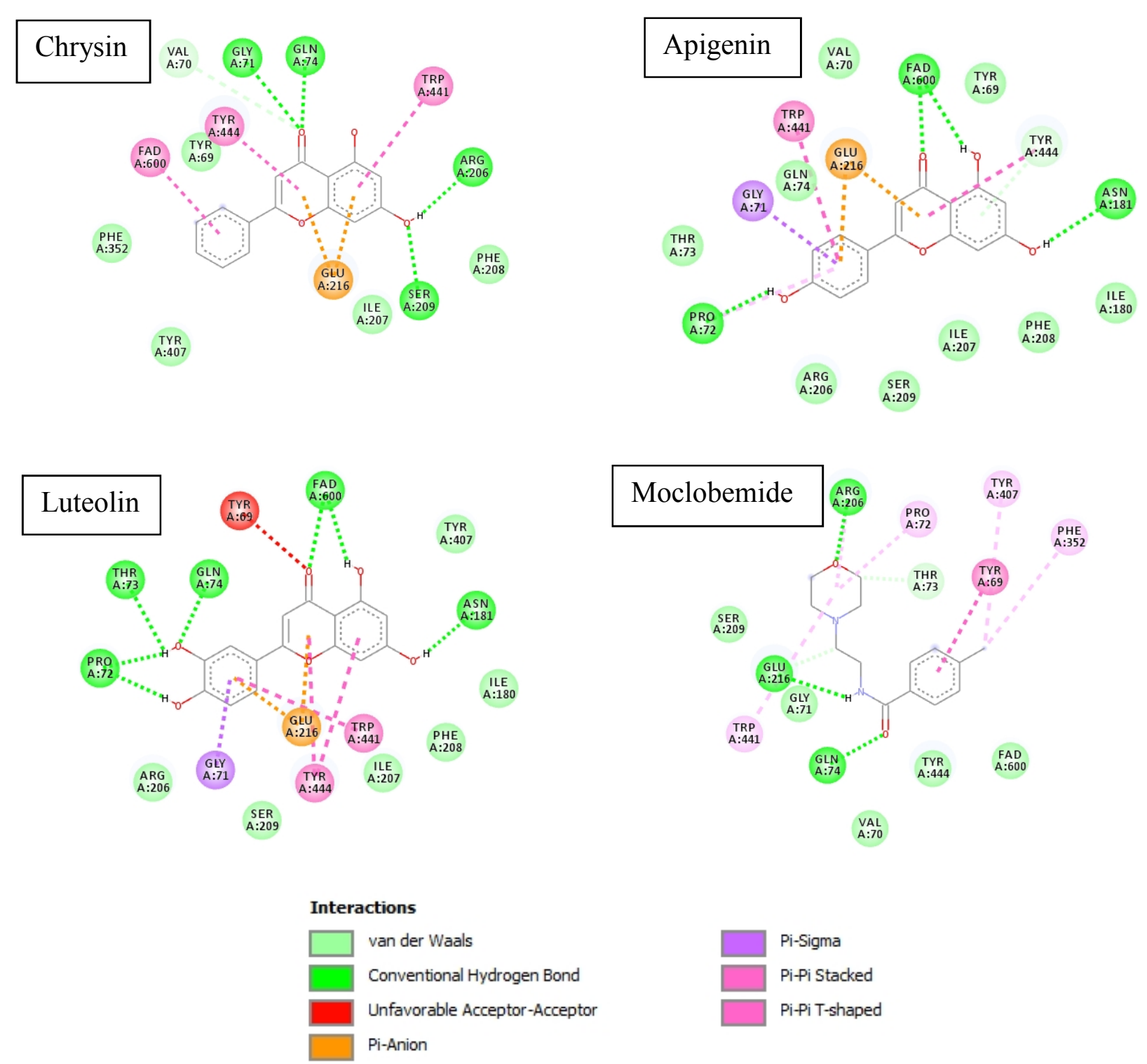

Fig. 2. 2D views of interacting complexes of ligand-receptor.

\section{Conclusion}

The obtained results of this work based on the in silico MD simulations could show the efficacy of flavonoid ligands for MAO-A enzyme inhibitions, in which they could be summarized into three main trends. First, flavonoid derivatives could be proposed for MAO-A inhibition by their interactions with FAD coenzyme. Second, the binding strength of luteolin with three functionalized hydroxyl groups could be very well compared with the reference moclobemide with a lower BE value. Third, details of ligand-receptor interactions of flavonoid-MAO-A could be very well recognized by the in silico MD simulations, in which both of quantitative and qualitative properties are very much important for the final judgments about the complex interacting systems. For such in silico approaches, obtaining several detailed results is an advantage versus experimental works; therefore, in silico works could be performed prior to experiments for better experimental design.

\section{Acknowledgments}

The general supports by Isfahan University of Medical Sciences are gratefully acknowledged. 


\section{References}

1. D. Checknita, T.J. Ekstrom, E. Comasco, K.W. Nilsson, J. Tiihonen, S. Hodgins; Associations of monoamine oxidase A gene first exon methylation with sexual abuse and current depression in women. J. Neural Transmis. 125 (2018) 1053-1064.

2. R. Bennett, E. Blochouse, D. Leech; Effect of individual plasma components on the performance of a glucose enzyme electrode based on redox polymer mediation of a flavin adenine dinucleotide-dependent glucose dehydrogenase. Electrochim. Acta 302 (2019) 270-276.

3. R.B. Katz, M. Toprak, S.T. Wilkinson, G. Sanacora, R. Ostroff; Concurrent use of ketamine and monoamine oxidase inhibitors in the treatment of depression: A letter to the editor. Gen. Hospital Psychiat. 54 (2018) 6264.

4. G. Li, H.X. Lou; Strategies to diversify natural products for drug discovery. Med. Res. Rev. 38 (2018) 1255-1294.

5. S. Mushtaq, B.H. Abbasi, B. Uzair, R. Abbasi; Natural products as reservoirs of novel therapeutic agents. EXCLI J. 17 (2018) 420451.

6. S.H. Nile, Y.S. Keum, A.S Nile, S.S. Jalde, R.V. Patel; Antioxidant, anti-inflammatory, and enzyme inhibitory activity of natural plant flavonoids and their synthesized derivatives. J. Biochem. Mol. Toxicol. 32 (2018) e22002.

7. X.Y. Liu, X. Lv, P. Wang, C.Z. Ai, Q.H. Zhou, M. Finel, B. Fan, Y.F. Cao, H. Tang, G.B. Ge; Inhibition of UGT1A1 by natural and synthetic flavonoids. Int. J. Biol. Macromol. 126 (2019) 653-661.

8. M. Soleimani, M. Mirzaei, M.R. Mofid, G. Khodarahmi, S.F. Rahimpour; Lactoperoxidase inhibition by tautomeric propylthiouracils. Asian J. Grenn Chem. 4 (2020) 1-10.
9. T. Partovi, M. Mirzaei, N.L. Hadipour; The C$\mathrm{H} \cdots \mathrm{O}$ hydrogen bonding effects on the 170 electric field gradient and chemical shielding tensors in crystalline 1-methyluracil: A DFT study. Z. Naturforsch. A 61 (2006) 383-388.

10. M. Mirzaei, M. Meskinfam; Computational NMR studies of silicon nanotubes. Comput. Theor. Chem. 978 (2011) 123-125.

11. M. Mirzaei; Uracil-functionalized ultra-small $(n, 0)$ boron nitride nanotubes $(n=3-6)$ : Computational studies. Superlat. Microstruct. 57 (2013) 44-50.

12. M. Mirzaei; Effects of carbon nanotubes on properties of the fluorouracil anticancer drug: DFT studies of a CNT-fluorouracil compound. Int. J. Nano Dimens. 3 (2013) 175-179.

13. M. Mirzaei, R.S. Ahangari; Formations of CNT modified 5-(halogen) uracil hybrids: DFT studies. Superlat. Microstruct. 65 (2014) 375379

14. E. Naderi, M. Mirzaei, L. Saghaie, G. Khodarahmi, O. Gulseren; Relaxations of methylpyridinonetautomers at the $\mathrm{C60}$ surfaces: DFT studies. Int. J. Nano Dimens. 8 (2017) 124-131.

15. M. Mirzaei, N.L Hadipour; A computational NQR study on the hydrogen-bonded lattice of cytosine-5-acetic acid. J. Comput. Chem. 29 (2008) 832-838.

16. O.M. Ozkendir, M. Mirzaei; Alkali metal chelation by 3-hydroxy-4-pyridinone. Adv. J. Chem. B 1 (2019) 10-16.

17. K.E. Hayes, P. Batsomboon, W.C. Chen, B.D. Johnson, A. Becker, S. Eschrich, Y. Yang, A.R. Robart, G.B. Dudley, W.J. Geldenhuys, L.A. Hazlehurst; Inhibition of the FAD containing ER oxidoreductin 1 (Ero1) protein by EN-460 as a strategy for treatment of multiple myeloma. Bioorg. Med. Chem. 27 (2019) 1479-1488. 
18. P.A. Ghamsari, M. Samadizadeh, M. Mirzaei; Cytidine derivatives as inhibitors of methyltransferase enzyme. Eurasian Chem. Commun. 1 (2019) 310-317.

19. L. Chiuccariello, R.G. Cooke, L. Miler, R.D. Levitan, G.B. Baker, S.J. Kish, N.J. Kolla, P.M. Rusjan, S. Houle, A.A. Wilson, J.H. Meyer; Monoamine oxidase-A occupancy by moclobemide and phenelzine: implications for the development of monoamine oxidase inhibitors. Int. J. Neuropsychopharmacol. 19 (2016) 1-9.

20. H. Nazemi, M. Mirzaei, E. Jafari; Antidepressant activity of curcumin by monoamine oxidase-A inhibition. Adv. J. Chem. B 1 (2019) 3-9.
21. H.E. Pence, A. Williams; ChemSpider: an online chemical information resource, 2010.

22. P.W. Rose, A. Prlić, A. Altunkaya, C. Bi, A.R. Bradley, C.H. Christie, et al.; The RCSB protein data bank: integrative view of protein, gene and 3D structural information. Nucleic Acids Res. 45 (2017) D271-D281.

23. G.M. Morris, R. Huey, W. Lindstrom, M.F. Sanner, R.K. Belew, D.S. Goodsell, A.J. Olson; Autodock4 and AutoDockTools4: automated docking with selective receptor flexiblity. J. Computat. Chem. 16 (2009) 2785-2791.

24. Z.S. Alidoosti, M. Mirzaei; Comparative examination of moclobemide, tranylcypromine, phenelzine and isocarboxazid for monoamine oxidase-A inhibition. Adv. J. Chem. B 1 (2019) 23-28. 